Li, S., Wakefield, J. \& Noble, J. A. (2011). 'Automated segmentation and alignment of mitotic nuclei for Kymograph visualisation'. In: Proceedings of the 2011 8th IEEE International Symposium on Biomedical Imaging: From Nano to Micro, 30 March- 2 April 2011, Chicago, IL, USA, Institute of Electrical and Electronic Engineers, pp. 622-625. (c) 2011 by the Institute of Electrical and Electronics Engineers, Inc. Available at http://dx.doi.org/10.1109/ISBI.2011.5872484

\title{
AUTOMATED SEGMENTATION AND ALIGNMENT OF MITOTIC NUCLEI FOR KYMOGRAPH VISUALISATION
}

\author{
Simon Li $\quad$ James Wakefield ${ }^{\star} \quad$ J Alison Noble ${ }^{\star}$ \\ *Institute of Biomedical Engineering, Dept of Engineering Science, University of Oxford, OX3 7DQ \\ ${ }^{\dagger}$ College of Life and Environmental Sciences, University of Exeter, EX4 4QD
}

\begin{abstract}
The mechanics of many biological processes can only be uncovered through the analysis of spatio-temporal data. Kymographs are a popular tool for visualising dynamic processes whose movements can be mapped into a single dimension, such as mitosis, or cell division. However, global movements of a cell means the region of interest (ROI) used to create the kymograph must move with each frame. Here we present an automated algorithm for the selection of dynamic ROIs to allow the construction of kymographs of proteins associated with the mitotic spindle in Drosophila embryos. We segment a dividing nucleus using a level set coupled to a shape model, which we then use to align the segmented nuclei to a common frame of reference.
\end{abstract}

Index Terms - Cells (biology), Microscopy, Image segmentation, Visualisation

\section{INTRODUCTION}

Mitosis is the process through which cells replicate their DNA and divide into two daughter cells. It involves a highly orchestrated sequence of processes which are regulated by a complex network of interacting proteins whose roles may include initiation and modulation of subprocesses, blocking to prevent premature activation of required proteins, and monitoring to ensure all stages of mitosis proceed correctly and without error. Failure to correctly regulate this process is one of the major factors leading to tumourigenesis, and ultimately cancer.

New imaging and labelling technologies have enabled biologists to visualise in real time the dynamics of many of these vital mitotic proteins, but the intricate network of interactions has complicated attempts to probe the functions of individual proteins. Typically the function of a protein is inferred by perturbing the system, for instance by partially or fully inactivating the protein of interest, but this frequently leads to catastrophic failure of cell division, indicating the importance of that protein but providing little information on the underlying mechanics which led to failure.

This work is funded by an EPSRC Doctoral Training Grant number EP/E501605/1.
There is therefore interest in using automated and semiautomated imaging tools to quantify the change in dynamics of proteins and subcellular structures in order to identify small but significant differences following a minor perturbation of the system. For instance, the automated tracking of the two centrosomes which form the poles of the mitotic spindle in Drosophila melanogaster embryos uncovered a vital role for the protein Akt when it was discovered that the two poles did not line up opposite each other as in normal embryos [1].

Automated tracking and measurement tools can provide vast amounts of quantitative information, particularly when we have a well defined, albeit difficult, problem. In other cases, such as when are are probing the function of an uncharacterised protein, cell biologists have unrivalled intuition and can use their expert knowledge to infer the hidden behaviour of the protein, and to generate hypotheses which can be tested using these automated tools. This leads to the question of how this information should be visualised, particularly when it is derived from temporal data.

Kymographs, or time-intensity plots, are one way of visualising the dynamics of mitosis. A kymograph projects a 2D or 3D region of interest (ROI) onto a single dimension, and changes over time can then be visualised as a 2D surface plot [2]. Many of the changes in mitosis occur parallel to the axis of the mitotic spindle making a kymograph an ideal way of displaying them, for example the separation of chromosomes in anaphase or the growth of microtubules from the spindle poles towards the centre. Experimental limitations frequently result in poor quality images where these objects of interest do not have a well defined boundary and segmentation is very difficult, so the aggregation of image data over one or more dimensions can significantly improve the visualisation of the dynamics of the labelled objects.

It may be possible to use fixed ROIs which don't move over time, however in practice global movements of dividing nuclei often occur which means the axis along which the projection is made may no longer coincide with the objects of interest. This has motivated the development of methods for creating dynamic ROIs, for instance by introducing additional markers which track the spindle as it moves.

Here we present an alternative method which involves segmenting the spindle, followed by the calculation of a 
frame of reference for the spindle and the creation of an ROI for the kymograph.

\section{BIOLOGICAL DATA}

Drosophila (fruit fly) embryos are a popular tool for studying mitosis. They are relatively easy to handle, and during the early stages of development the nuclei are spread over the cortex (surface) of the embryo, and divide synchronously and rapidly, approximately every 20 minutes, allowing large numbers of nuclei to be imaged.

We are interested in a protein complex called the chromosomal passenger complex (CPC) which undergoes large changes in localisation throughout mitosis, and plays a vital role in ensuring chromosomes are segregated correctly into the two daughter nuclei [3]. The members of this complex have been tagged with a green fluorescent protein (GFP) which is endogenously expressed, allowing us to view the dynamics of the CPC in-vivo. During early mitosis (prophase to metaphase) it primarily localises to the chromosome arms, particularly the kinetochores which are the points of attachment of the mitotic spindle, before translocating to the central spindle in late mitosis (anaphase) when the pairs of chromosomes separate to opposite poles of the spindle.

Importantly for our work it also shows a faint spindle localisation, and this enables us to analyse the dynamics of the complex without the need for an additional marker. If necessary a spindle marker such as $\alpha$-tubulin could be used, however increasing the number of live fluorescent markers is often difficult and increases the likelihood of unwanted side effects, particularly when observing a protein complex involved in regulatory processes, so minimising the number of required markers is desirable. Images such as the ones shown in Figure 1 were acquired on a Zeiss 510 Meta laser scanning confocal microscope.

\section{DETECTION OF NUCLEI}

At metaphase the CPC labelled nuclei appear as faint elliptical regions corresponding to the spindle localisation of the CPC (Figure 1b), with a line of bright dots along the short axis indicating the kinetochores which are found at the centre of each pair of chromosomes, embedded in the autofluorescent cytoplasm of the embryo. We first blur the image by filtering with a circular structuring element, then dilate the resulting image with the same structuring element. This results in an image with flat plateaus at the approximate location of each nucleus, which can be found by searching for all local maxima. The size of the structuring element is set so that only one local maxima is found per nucleus without overlapping a neighbouring nucleus. In practice the detection stage is relatively insensitive to the exact size, so we set it to around half the expected size of a nucleus.

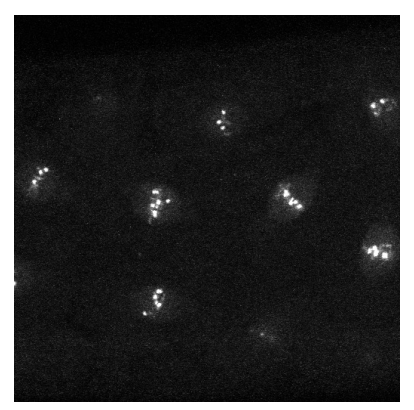

(a)

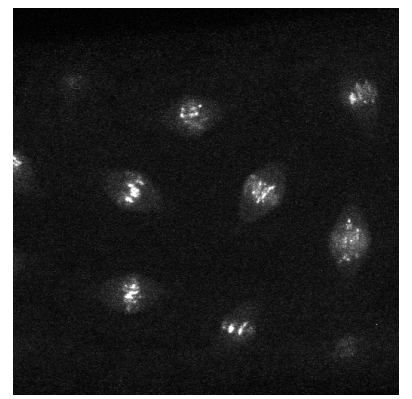

(c)

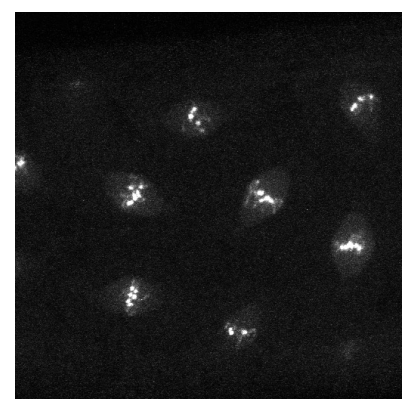

(b)

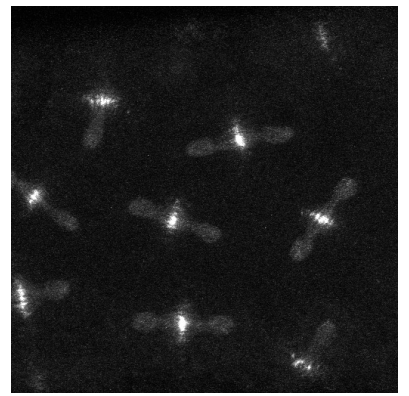

(d)
Fig. 1. Stills from a time lapse movie of dividing nuclei in a Drosophila embryo. (a) $0 \mathrm{sec}$ (b) $60 \mathrm{sec}$ (c) $90 \mathrm{sec}$ (d) 175 sec.

Occasionally a false detection is made, typically when the cytoplasm shows more auto-fluoresence than normal. In addition since the cortex is curved whereas the imaging plane is flat some nuclei may be out of focus and of low intensity. We iteratively discard any local maximum whose average intensity is less than $\mu-1.5 \sigma$ where $\mu$ and $\sigma$ are the mean and standard deviation of the mean intensities of all remaining local maxima. The results of the detection step are shown in Figure 2a.

\section{SEGMENTATION}

Our aim is to segment a nucleus based on the faint spindle fluorescence, so to reduce the noise we apply the Poisson total variation denoising algorithm of [4] which finds the denoised image $I_{n}(\mathbf{x})$ by minimising the energy:

$$
E=\int_{\Omega}\left|\nabla I_{n}(\mathbf{x})\right|+\frac{\lambda}{2} \int_{\Omega}\left(I_{n}(\mathbf{x})-I(\mathbf{x}) \log I_{n}(\mathbf{x})\right)
$$

where $I(\mathbf{x})$ is the observed image, $\Omega$ is the image domain and $\lambda$ controls the amount of regularisation.

We then apply a sigmoidal filter to adjust the intensity levels of the denoised image $I_{n}(\mathbf{x})$ by reducing the apparent brightness of the kinetochores

$$
I_{f}(\mathbf{x})=1 /\left(1+e^{-\left(I_{n}(\mathbf{x})-b\right) / a}\right)
$$


where $a$ controls the range of intensities which we wish to emphasize, and $b$ the midpoint of the intensity range. $a$ is set to $0.02 \times \max \left\{I_{n}(\mathbf{x})\right\}$ and $b$ to the median intensity of $I_{n}(\mathbf{x})$.

Our segmentation is based on the fast level sets algorithm developed by [5], which gives a pixel-level segmentation, together with the addition of a shape model following the method in [6]. Typical level set algorithms involve the time consuming solution of a partial differential equation which could give a sub-pixel solution, but since the noise levels in the images are quite high this would not offer improved accuracy.

This fast level set algorithm is essentially the ultimate narrowband method, taking into consideration only the pixels immediately either side of the contour. These pixels are placed into two lists, one for the inner edge of the contour and one for the outer edge. Every iteration of the level set involves moving each pixel on the boundary in or out by one pixel depending on the value of the speed field, of which only the sign (positive or negative) is considered. Regularisation of the contour is done by filtering the contour with a Gaussian kernel.

The signal-to-noise ratio of the images remains low, and the spindle fluorescence frequently fades out instead of having a well defined edge, so a shape model is used to prevent the level set from expanding too far. The exact shape of the spindle varies over time and since we might wish to apply this algorithm to other proteins with a similar though non-identical localisation we choose a simple model for the shape of the spindle, namely an ellipse, which we fit using the method of moments to find the ellipse whose second moments are the same as those of the segmented region. The shape model also incorporates an area prior which encourages the area of the segmented region to remain the same or increase slightly, in accordance with the observed behaviour of the spindle.

The initialisation for the first frame is provided by the detection stage, with a frame at time $t$ being initialised using the segmention from frame $t-1$.

The lack of edges between the background and spindle fluorescence means a region-based potential field must be used to govern the evolution of the level set [7]. This is modified slightly by using the local hybrid level set from [8] which considers a neighbourhood of radius $r$ around each point on the boundary for the region calculation.

\section{ALIGNMENT}

The segmentations for each nucleus could be used to obtain quantitative measurements, for example spindle length, area, and rate of movement. However we are interested in visualising the uncharacterised and often unclear behaviour of a protein occuring within the spindle region, and the following sections describe our approach.

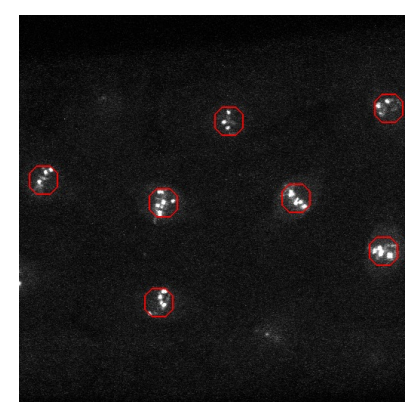

(a)

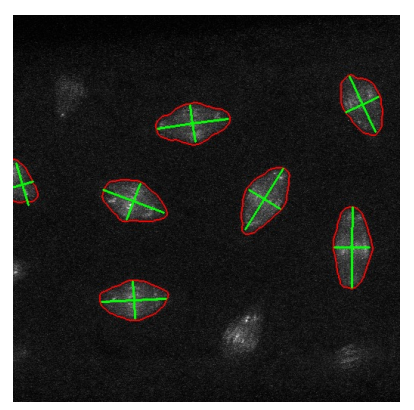

(b)
Fig. 2. (a) The results of the nuclei detection step on the first frame. (b) An example of a segmented frame, together with the major and minor axes of the ellipse model. Note that the nuclei have moved since the first frame.

The fitted ellipses used for each frame of the segmentation can be used to find a reference frame for the spindle in late mitosis, since the spindle displays a strongly elongated shape corresponding to the long axis of the ellipse, as shown in Figure 2b. During early mitosis the spindle region is circular, so the orientation of the ellipse at this stage is not useful.

A rotation operation is performed to bring all frames of all nuclei into a common orientation, and blank padding is added around the edges so that all images have the same dimensions. This is a useful visualisation tool for biologists, since it is now possible to simultaneously compare multiple nuclei, possibly from multiple embryos, by arranging all nuclei into a grid and successively viewing all temporal frames.

\section{KYMOGRAPHS}

Kymographs are typically created from a line or rectangular region, which may be positioned automatically by tracking point markers. However, the segmentation step means we can now create kymographs from irregularly shaped regions. We define the main spindle axis as the $x$-axis, and the $y$-axis as perpendicular, with the origin situated at the centre of the segmentation.

The kymograph is created by summing intensities along the $y$-axis, which results in a 1D intensity plot as a function of position $x$ along the spindle:

$$
k_{n}(x, t)=\sum_{y} J_{n, t}(x, y)
$$

where $J_{n, t}$ is one of the segmented and rotated nuclei from frame $t$. This is repeated for subsequent frames to form the complete kymograph $k_{n}(x, t)$ for nucleus $n$, an example of which is shown in Figure 3. The removal of one dimension helps to emphasize trends in the protein dynamics which are difficult to detect in the original images, such as the presence of multiple distinct localisations as opposed to a single widely 

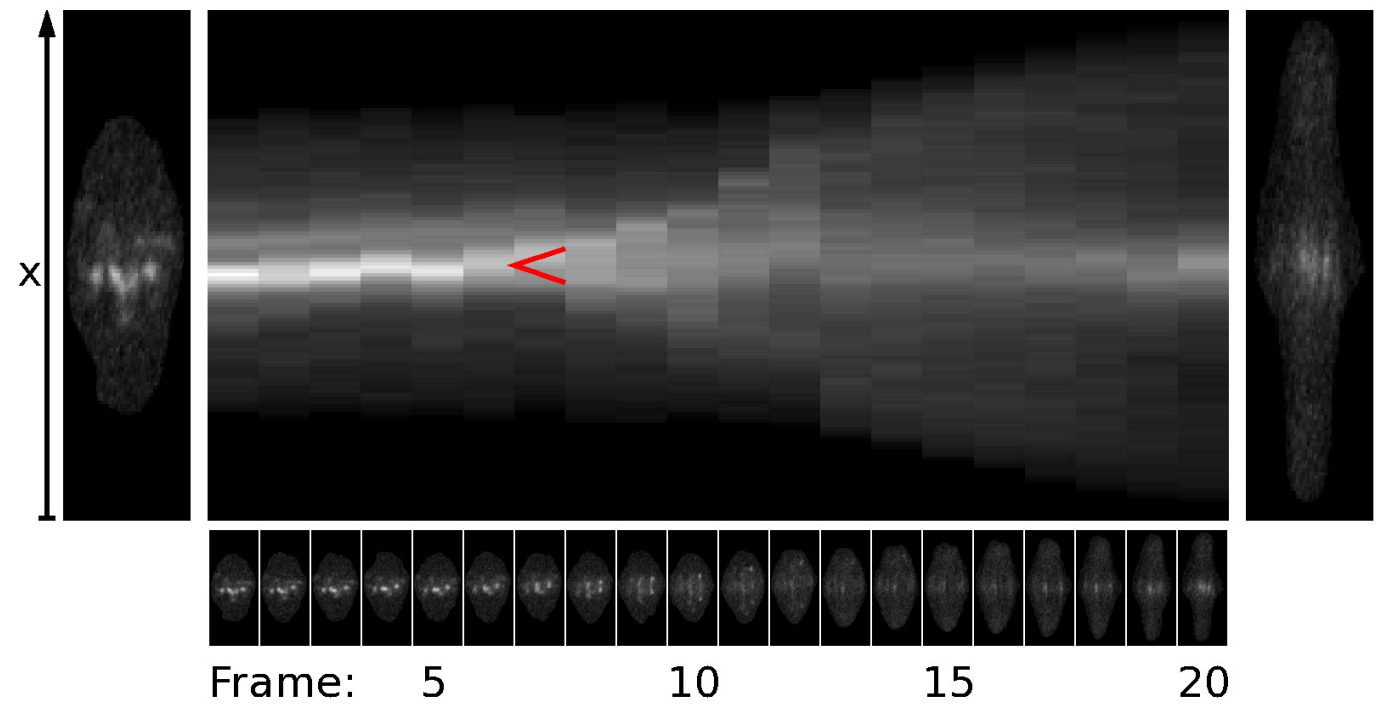

Fig. 3. A kymograph created from one segmented and aligned nucleus. The main spindle axis $x$ is plotted vertically, and the time axis horizontally. The first and last time frames used to form the kymograph are shown on the left and right. The $<$ symbol marks entry into anaphase, with the single central localisation dividing into three bands corresponding to the separating pairs of kinetochores, and the central spindle.

spread distribution, and the compact representation of temporally varying data would facilitate the comparison of different samples.

\section{CONCLUSIONS}

We have presented a method for automatically segmenting and visualising the dynamics of proteins associated with the mitotic spindle. Our segmentation and alignment steps allow multiple nuclei to be visualised synchronously, enabling us to efficiently utilise the expert knowledge of cell biologists to pick up small though potentially significant patterns and differences in the data. The use of kymographs as a method for summarising multi-dimensional spatio-temporal data in a single plot provides another way of visualising dynamic changes within and between nuclei, and is particularly valuable when the subcellular localisation of a labelled object has poorly defined boundaries.

\section{REFERENCES}

[1] Graham J Buttrick, Luke M A Beaumont, Jessica Leitch, Christopher Yau, Julian R Hughes, and James G Wakefield, "Akt regulates centrosome migration and spindle orientation in the early drosophila melanogaster embryo," J. Cell Biol., vol. 180, no. 3, pp. 537-48, 2008.

[2] António J Pereira and Helder Maiato, "Improved kymography tools and its applications to mitosis," Methods, vol. 51, no. 2, pp. 214-9, 2010.
[3] Sandrine Ruchaud, Mar Carmena, and William C Earnshaw, "Chromosomal passengers: conducting cell division,” Nat. Rev. Mol. Cell Biol., vol. 8, no. 10, pp. 798812, 2007.

[4] Triet Le, Rick Chartrand, and Thomas J. Asaki, "A variational approach to reconstructing images corrupted by poisson noise," Journal of Mathematical Imaging and Vision, vol. 27, no. 3, pp. 257, 2007.

[5] Yonggang Shi and William Clem Karl, "A real-time algorithm for the approximation of level-set-based curve evolution," IEEE transactions on image processing, vol. 17, no. 5, pp. 645-56, 2008.

[6] Christopher Yau and James G Wakefield, "Quantitative image analysis of chromosome dynamics in early drosophila embryos," in 2007 4th IEEE International Symposium on Biomedical Imaging From Nano to Macro, 2007, p. 264.

[7] T F Chan and L A Vese, "Active contours without edges," IEEE transactions on image processing, vol. 10, no. 2, pp. 266-77, 2001.

[8] Shawn Lankton, Delphine Nain, Anthony Yezzi, and Allen Tannenbaum, "Hybrid geodesic region-based curve evolutions for image segmentation," in Proceedings of SPIE, 2007, vol. 6510, p. 65104U. 